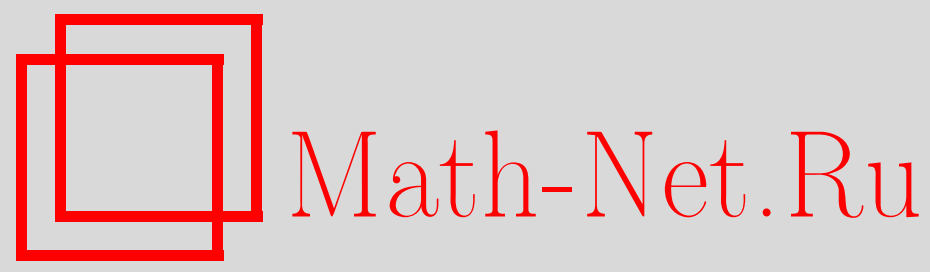

Ю. Я. Агранович, О. Т. Азизова, О связи смешанных дискриминантов и совместного спектра семейства коммутирующих операторов в конечномерном пространстве, Матем. заметки, 1997, том 62, выпуск 1, 3-9

DOI: https://doi.org/10.4213/mzm1582

Использование Общероссийского математического портала Math-Net.Ru подразумевает, что вы прочитали и согласны с пользовательским соглашением http://www . mathnet.ru/rus/agreement

Параметры загрузки:

IP: 54.89 .56 .158

26 апреля 2023 г., 17:08:22

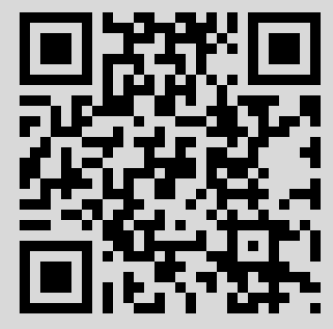




\title{
О СВЯЗИ СМЕШАННЫХ ДИСКРИМИНАНТОВ \\ И СОВМЕСТНОГО СПЕКТРА \\ СЕМЕЙСТВА КОММУТИРУЮШИХ ОПЕРАТОРОВ \\ В КОНЕЧНОМЕРНОМ ПРОСТРАНСТВЕ
}

\author{
Ю.Я. Агранович, О. Т. Азизова
}

Исследуются свойства полиномиального операторного пучка

$$
L(\lambda)=\sum_{i=0}^{n} \lambda^{n-i} M_{i}, \quad M_{i}: \mathscr{H} \rightarrow \mathscr{H}, \quad i=\overline{0, n}
$$

$\mathscr{H}-k$-мерное гильбертово пространство. Доказано, что смешанные дискриминанты $\left\{d_{j}\right\}_{j=0}^{n k}-$ коэфффициенты полинома

$$
\operatorname{det} L(\lambda)=\sum_{j=0}^{n k} d_{j} \lambda^{n k-j}
$$

- определяются только совместньм спектром семейства $\left\{M_{i}\right\}_{i=0}^{n}$. Получено обобщение известной теоремы Гершгорина о локализации собственных значений матрицы на случай полиномиального матричного пучка.

Библиограффия: 9 названий.

Предметом нашего рассмотрения будет полиномиальньй операторный пучок вида

$$
L(\lambda)=\sum_{i=0}^{n} \lambda^{n-i} M_{i}
$$

с коэффициентами $\left\{M_{i}\right\}_{i=0}^{n}$, являющимися операторами в конечномерном комплексном гильбертовом пространстве $\mathscr{H}(\operatorname{dim} \mathscr{H}=k)$. Понятно,что $\operatorname{det} L(\lambda)$ представляет собой некоторый многочлен, степень которого не превосходит $n k$ :

$$
\operatorname{det} L(\lambda)=\sum_{i=0}^{n k} d_{i} \lambda^{n k-i}
$$

Коэффициенты $\left\{d_{i}\right\}_{i=0}^{n k}$ в $(2)$ - смешанные дискриминанты, построенные по семейству матричных представлений операторных коэффициентов в (1), - впервые появились, 
по-видимому, в работах Минковского и Вейля, а также Боннезена и Фенхеля, и систематически применялись Александровым (см. [1]) в его теории смешанных объемов вьпуклых тел.

Из (1) и (2) следует, что величины $\left\{d_{i}\right\}_{i=0}^{n k}$ инвариантны относительно действия любого унитарного автоморфизма на $L(\lambda)$. Однако, до сих пор неизвестны необходимые и достаточные условия, которым должны удовлетворять операторные коэффициенты $\mathrm{M}_{j}$ $(j=\overline{0, n})$ полиномиального пучка $(1)$, чтобы коэффициенты $d_{i}(i=\overline{0, n k})$ многочлена (2) зависели только от инвариантов операторов $\left\{M_{j}\right\}_{j=0}^{n}$.

Цель настоящей работы - доказать, что если семейство $\left\{M_{j}\right\}_{j=0}^{n}$ коммутативно, то коэффициенты $\left\{d_{i}\right\}_{i=0}^{n k}$ однозначно определяются координатами точек совместного спектра $\sigma\left(\left\{M_{j}\right\}_{j=0}^{n}\right)$ операторных коэффициентов и их кратностями. Полученные результаты применяются в задаче о локализации собственных значений пучка (1): продолжая работу [2], где этот вопрос изучался в терминах смешанных дискриминантов, мы докажем некоторое обобщение известной теоремы Гершгорина (см., например, [3, с. 58-71]).

1. Рассмотрим полиномиальньй операторный пучок

$$
L(\lambda)=\sum_{i=0}^{n} \lambda^{n-i} A_{i}, \quad A_{i}: \mathscr{H} \rightarrow \mathscr{H}, \quad i=\overline{0, n}
$$

Пусть семейство операторов $\left\{A_{i}\right\}_{i=0}^{n}$ содержится в некоторой коммутативной алгебре $\mathfrak{S}$ операторов скалярного типа, действующих в $\mathscr{H}$. Тогда (см., например, [4, с. 113]) существует оператор $A$, также скалярного типа, и $n+1$ многочленов $p_{i}$, степени которых меньше степени минимального аннулирующего многочлена для $A$, таких, что

$$
A_{i}=p_{i}(A), \quad i=\overline{0, n}
$$

Обозначим через $\mathfrak{U}$ совокупность всех унитарньх операторов $U: \mathscr{H} \rightarrow \mathscr{H}$ таких, что действие соответствующих автоморфизмов $U^{*} A U$ приводит оператор $A$ (терминологию см. в [5, с. 97]). Из (4) немедленно следует, что каждьй элемент $U \in \mathfrak{U}$ определяет унитарньй автоморфизм, приводящий сразу все $\left\{A_{i}\right\}(i=\overline{0, n})$. При этом порядок расположения собственных чисел оператора $A$ на главной диагонали соответствующего матричного представления $U^{*} A U$ определяет порядок расположения собственных чисел матричного представления операторов $U^{*} A_{i} U(i=\overline{0, n})$. Иными словами, каждому элементу $U \in \mathfrak{U}$ мы можем поставить в соответствие множество точек в $\mathbb{C}^{n+1}$ :

$$
\begin{gathered}
U^{*} A_{i} U=\operatorname{diag}\left(a_{l}^{(i)}\right), \quad l=\overline{1, k}, \quad i=\overline{0, n}, \\
U \mapsto\left\{\left\{a_{l}^{(i)}\right\}_{i=0}^{n}\right\}_{l=1}^{k} \equiv \tau\left(\left\{A_{i}\right\}_{i=0}^{n}, U\right) \subset \mathbb{C}^{n+1} .
\end{gathered}
$$

Если $U_{1}, U_{2} \in \mathfrak{U}$, то из (4) следует, что $\tau\left(\left\{A_{i}\right\}_{i=0}^{n}, U_{1}\right)=\tau\left(\left\{A_{i}\right\}_{i=0}^{n}, U_{2}\right)$. Таким образом, все элементы $\mathfrak{U}$ определяют одно и то же множество точек

$$
\tau\left(\left\{A_{i}\right\}_{i=0}^{n}, \mathfrak{U}\right) \equiv \tau\left(\left\{A_{i}\right\}_{i=0}^{n}\right) \subset \mathbb{C}^{n+1} .
$$

Пусть множество $\tau\left(\left\{A_{i}\right\}_{i=0}^{n}\right)$ состоит из $m$ различных точек, $j$-я из которых встречается в $(5) n_{j}$ раз $(j=\overline{1, m})$, тогда $\sum_{j=1}^{m} n_{j}=k$, и мы можем сформулировать следующее 
ОПРЕДЕЛЕНИЕ 1. Построенное в (5) множество $\tau\left(\left\{A_{i}\right\}_{i=0}^{n}\right) \subset \mathbb{C}^{n+1}$ будем назьвать совместным спектром семейства операторов $\left\{A_{i}\right\}_{i=0}^{n} \subset \mathfrak{S}$, а числа $\left\{n_{j}\right\}_{j=0}^{m}$ будем назьвать кратностями соответствуюших различных точек совместного спектра.

На самом деле, определение совместного спектра совпадает с абстрактным определением для коммутативной банаховой алгебры (см., например, [6, с. 267]) в рассматриваемом нами случае алгебры $\mathfrak{S}$.

Действительно, зафиксируем любую точку $\left\{a_{j}^{(i)}\right\}_{i=0}^{n} \in \tau\left(\left\{A_{i}\right\}_{i=0}^{n}\right)$ и произвольное семейство операторов $\left\{B_{i}\right\}_{i=0}^{n} \subset \mathfrak{S}$. Рассмотрим выражение

$$
M=\sum_{i=0}^{n}\left(A_{i}-a_{j}^{(i)} I\right) B_{i} .
$$

Приводя все $\left\{A_{i}\right\}_{i=0}^{n}$ и переходя к соответствующему матричному представлению, с учетом (5) немедленно получим, что $M_{j j}=0$, следовательно, $M \neq I$. Таким образом, множество $\tau\left(\left\{A_{i}\right\}_{i=0}^{n}\right)$ содержится в совместном спектре $\sigma\left(\left\{A_{i}\right\}_{i=0}^{n}\right)$ в смысле абстрактного определения. Докажем обратное включение. Если $\left\{\psi^{(i)}\right\}_{i=0}^{n} \in \sigma\left(\left\{A_{i}\right\}_{i=0}^{n}\right)$, то для любых $\left\{B_{i}\right\}_{i=0}^{n} \subset \mathfrak{S}$ оператор

$$
M_{1}=\sum_{i=0}^{n}\left(A_{i}-\psi^{(i)} I\right) B_{i}
$$

вырожден, в противном случае мы могли бы домножить обе части последнего равенства на $M_{1}^{-1}$ и получили бы

$$
\sum_{i=0}^{n}\left(A_{i}-\psi^{(i)} I\right) B_{i} M_{1}^{-1}=I
$$

что противоречит абстрактному определению совместного спектра. Приведем $M_{1}$ действием унитарного автоморфизма, определяемого некоторьп элементом $U \in \mathfrak{U}$. Выберем $B_{i}$ так, чтобы матричное представление $U^{*} B_{i} U$ было диагональным для всех $i=\overline{0, n}$. В силу абстрактного определения совместного спектра $B_{i}$ выбираются произвольным образом, и, следовательно, мы имеем возможность подходящим образом варьировать диагональные элементы матричных представлений $\left\{U^{*} B_{i} U\right\}_{i=0}^{n}$. Отсюда непосредственно вытекает, что если $M_{1}$ вырожден, то в одной и той же позиции диагональных матрищ $A_{i}-\psi^{(i)} I$ должен стоять 0 , т.е. $\psi^{(i)}=a_{j}^{(i)}(i=\overline{0, n})$ для некоторой точки $\left\{a_{j}^{(i)}\right\}_{i=0}^{n} \in \tau\left(\left\{A_{i}\right\}_{i=0}^{n}\right)$. Тем самым, доказано обратное включение, следовательно, $\tau\left(\left\{A_{i}\right\}_{i=0}^{n}\right)=\sigma\left(\left\{A_{i}\right\}_{i=0}^{n}\right)-$ совместный спектр в смысле абстрактного определения.

Переходя теперь к матричному представлению $\left\{A_{i}\right\}_{i=0}^{n}$, из $(5),(2)$ и (3) получим соотношение

$$
\operatorname{det} L(\lambda)=\prod_{j=1}^{m}\left(\sum_{i=0}^{n} a_{j}^{(i)} \lambda^{n-i}\right)^{n_{j}}
$$

Таким образом, доказана 
ЛЕмма 1. Пусть $\left\{A_{i}\right\}_{i=0}^{n}-$ произвольное конечное семейство операторов из коммутативной алгебры $\mathfrak{S}$ операторов скалярного типа, действуюших в $k$-мерном гильбертовом пространстве $\mathscr{H}$. Пусть $\sigma\left(\left\{A_{i}\right\}_{i=0}^{n}\right)=\left\{\left\{a_{j}^{(i)}\right\}_{i=0}^{n}\right\}_{j=1}^{m}-$ совместный спектр операторов $\left\{A_{i}\right\}_{i=0}^{n}$, состоящий из $m$ различных точек кратностей $n_{j}(j=\overline{1, m})$. Тогда смешанные дискриминанты $\left\{d_{i}\right\}_{i=0}^{n k}$ в $(2)$ однозначно определяются координатами точек совместного спектра операторов $\left\{A_{i}\right\}_{i=0}^{n} u$ их кратностями:

$$
\sum_{i=0}^{n k} d_{i} \lambda^{n k-i}=\prod_{j=1}^{m}\left(\sum_{i=0}^{n} a_{j}^{(i)} \lambda^{n-i}\right)^{n_{j}}
$$

СледСТВИЕ 1. Пусть $\left\{A_{i}\right\}_{i=0}^{n}$ - произвольное конечное семейство операторов из алгебры самосопряженных операторов, действуюших в Н . Тогда оно удовлетворяет условиям леммы 1 и коэффициенты при степенях $\lambda$ в (7) вещественны.

ДокАЗАТЕЛьство. Так как самосопряженность произведения самосопряженных операторов эквивалентна их коммутативности, то остается применить лемму 1 и воспользоваться тем, что собственные числа самосопряженных операторов вещественны.

2. Рассмотрим полиномиальньй операторный пучок

$$
L(\lambda)=\sum_{i=0}^{n} \lambda^{n-i} C_{i}, \quad C_{i}: \mathscr{H} \rightarrow \mathscr{H}, \quad i=\overline{0, n}
$$

Пусть теперь семейство операторов $\left\{C_{i}\right\}_{i=0}^{n}$ принадлежит некоторой коммутативной алгебре $\mathfrak{M}$ операторов, необязательно скалярного типа, действующих в $\mathscr{H}$. В силу разложения Данфорда (см. [7, с. 31], а также, например, [5, с. 123-125]) имеем

$$
C_{i}=S_{i}+N_{i}, \quad i=\overline{0, n}
$$

где $S_{i}$ - операторы скалярного типа, а $N_{i}$ - нильпотентные операторы; $S_{i}$ и $N_{i}$ коммутируют и разложение (9) единственно. Из абстрактных результатов теории спектральных операторов следует, что $S_{i}$ и $S_{j}$ коммутируют $(i, j=\overline{0, n}, i \neq j)[7$, с. 27] и алгебра, порожденная семейством попарно коммутирующих операторов скалярного типа $\left\{S_{i}\right\}_{i=0}^{n}$, является подалгеброй алгебры $\mathfrak{M}$ (см. [7, с. 279]). Учитывая указанные обстоятельства и произвольность выбора алгебры коммутирующих операторов скалярного типа $\mathfrak{S}$ в п. 1 , мы, без ограничения общности, можем принять, что $S_{i}=A_{i}(i=\overline{0, n})$ и воспользоваться рассуждениями и результатами п. 1. Кроме того, известно (см., например, [8, c. 103]), что существует унитарный автоморфизм такой, что матричное представление $U^{*} C U$ имеет верхнетреугольньй вид сразу для всех операторов $C \in \mathfrak{M}$. Значит, мы можем полностью перенести определение 1 из п. 1 на рассматриваемый здесь случай и, кроме того, получить факторизационное представление (6) для пучка (8). Тем самьц, доказана

ТЕОремА 1. Пусть $\left\{C_{i}\right\}_{i=0}^{n}-$ произвольное конечное семейство операторов из коммутативной алгебры $\mathfrak{M}$ операторов, действующих в $k$-мерном гильбертовом пространстве $\mathscr{H}$. Пусть $\sigma\left(\left\{C_{i}\right\}_{i=0}^{n}\right)=\left\{\left\{c_{j}^{(i)}\right\}_{i=0}^{n}\right\}_{j=1}^{l}-$ совместный спектр операторов $\left\{C_{i}\right\}_{i=0}^{n}$, состоящий из l различных точек кратностей $n_{j}(j=\overline{1, l})$. Тогда 
смешанные дискриминанты $d_{i}(i=\overline{0, n k})$ в (2) однозначно определяются координатами точек совместного спектра операторов $\left\{C_{i}\right\}_{i=0}^{n}$ u их кратностями $u$ справедливо факторизачионное представление

$$
\sum_{i=0}^{n k} d_{i} \lambda^{n k-i}=\prod_{j=1}^{l}\left(\sum_{i=0}^{n} c_{j}^{(i)} \lambda^{n-i}\right)^{n_{j}}
$$

СлЕДСТВИЕ 2. Пусть выполнены условия теоремы 1. Тогда $\mathscr{H}$ разлагается в прямую сумму подпространств $\mathscr{L}_{j}(j=\overline{1, l}), \operatorname{dim} \mathscr{L}_{j}=n_{j}$, каждое из которых является собственным для всех $S_{i}$ и корневым для всех $C_{i}(i=\overline{0, n})$.

ДокАЗАТЕЛЬСТВо. Из рассуждений п. 1 ясно, что совокупность скалярных составляющих $S_{i}$ операторов $C_{i}$ имеет совместную систему собственных подпространств $\mathscr{L}_{j}$ и $\operatorname{dim} \mathscr{L}_{j}=n_{j}(j=\overline{1, l})$. Так как собственное подпространство составляющей скалярного типа $S_{i}$ является корневым подпространством для $C_{i}$, то и система корневых подпространств операторов $\left\{C_{i}\right\}_{i=0}^{n}$ также является для них совместной.

ЗАмечАниЕ 1. Утверждение, обратное следствию 2, конечно, неверно: соответствующие контрпримеры легко строятся с использованием матриц, имеющих нижне- и верхнетреугольную формы.

ЗАмЕчАнИЕ 2. Из наших построений ясно, что совместньй спектр полностью определяется составляющими скалярного типа, и, следовательно, формально совместньй спектр можно определить для некоммутируюших операторов спектрального типа: требуется лиш коммутирование составляющих скалярного типа. Однако, без условия коммутируемости $N_{i}$ и $N_{j}(i \neq j, i, j=\overline{0, n})$ результаты теоремы 1 , вообще говоря, неверны.

Теперь сформулируем полезные, как нам представляется, следствия.

СлЕДСТВИЕ 3. Пусть выполнены условия теоремы 1. Тогда собственные числа оператора $L(\lambda)$ и его след равны соответственно

$$
\mu_{j}=\sum_{i=0}^{n} c_{j}^{(i)} \lambda^{n-i}, \quad j=\overline{1, l} ; \quad \operatorname{sp} L(\lambda)=\sum_{j=1}^{l} n_{j}\left(\sum_{i=0}^{n} c_{j}^{(i)} \lambda^{n-j}\right)
$$

Доказательство непосредственно вытекает из следствия 2.

Аналогично, по известным формулам вычисляются и другие инварианты оператора $L(\lambda)$.

СлЕДСТВИЕ 4. Пусть выполнены условия теоремы 1. Тогда следующие условия әквивалентны:

1) $\sigma(L(\lambda)) \equiv \mathbb{C}$

2) $\theta \in \sigma\left(\left\{C_{i}\right\}_{i=0}^{n}\right)$.

Доказательство немедленно следует из теоремы 1. 
3. Полученные в п. 2 результаты позволяют продолжить исследования, начатыев [2], и доказать в терминах совместного спектра операторных коэффищиентов $\left\{C_{i}\right\}_{i=0}^{n}$ обобщение теоремы Гершгорина (см., например, [3, с. 58-71]) на случай полиномиальных операторных пучков. Если вьполнены условия

$$
c_{j}^{(0)} \neq 0, \quad j=\overline{1, l}
$$

то мы можем определить величины

$$
\gamma_{j}=2 \max _{i=\overline{1, n}}\left|\frac{c_{j}^{(i)}}{c_{j}^{(0)}}\right|^{1 / i}, \quad j=\overline{1, l}
$$

Если вьполнены условия

$$
c_{j}^{(n)} \neq 0, \quad j=\overline{1, l}
$$

то, аналогично, определим величины

$$
\Gamma_{j}=2 \max _{i=\overline{1, n}}\left|\frac{c_{j}^{(i)}}{c_{j}^{(n)}}\right|^{1 /(n-i)}, \quad j=\overline{1, l}
$$

Воспользовавшись теперь результатами Элснера [9], получим, что спектр пучка (8) $\sigma(L(\lambda))$ содержится в объединении $l$ колец $\Omega_{j}$, определяемых условиями

$$
\left(\sum_{i=1}^{n}\left|\frac{c_{j}^{(n-i)}}{c_{j}^{(n)}}\right| \cdot \Gamma_{j}^{i}\right)^{-1 / n} \frac{1}{\psi(n)} \leqslant|\lambda| \leqslant\left(\sum_{i=1}^{n}\left|\frac{c_{j}^{(i)}}{c_{j}^{(0)}}\right| \cdot \gamma_{j}^{n-i}\right)^{1 / n} \psi(n), \quad j=\overline{1, l}
$$

где

$$
\psi(n)= \begin{cases}n, & \text { если } n \text { нечетно, } \\ n-1, & \text { если } n \text { четно. }\end{cases}
$$

Таким образом, доказана

ТЕОРема 2. Пусть выполнены условия теоремы 1, (10) и (11). Тогда

$$
\sigma(L(\lambda)) \subset \bigcup_{j=1}^{l} \Omega_{j}(\equiv \Omega)
$$

где $\Omega_{j}$ определяются условиями (12).

СлЕДСТВИЕ 5. Если количество различных точек совместного спектра $\sigma\left(\left\{C_{i}\right\}_{i=0}^{n}\right)$ меньие $k$, то $\sigma(L(\lambda))$ содержит по крайней мере одно кратное собственное значение. Пусть одна из связных компонент $\Omega$ образована объединением $r$ колеи вида (12), тогда эта связная компонента содержит ровно $\mathrm{nr}$ собственных значений пучка $L(\lambda)$ с учетом их кратности. 


\section{СПИСОК ЦИТИРОВАННОЙ ЛИТЕРАТУРЫ}

[1] Александров А. Д. К теории смешанных объемов выпуклых тел // Матем. сб. 1938. T. 3(45). № 2. С. 227-249.

[2] Агранович Ю.Я., Сухочёва Л. И. О собственных значениях полиномиальных матричных пучков // Спектральные и эволюционные задачи. Киев: Наукова думка, 1991. С. 23.

[3] Пароди М. Локализация характеристических чисел матриц и ее применения. М.: ИЛ, 1960.

[4] Маркус М., Минк X. Обзор по теории матриц и матричных неравенств. М.: Наука, 1972.

[5] Глазман И. М., Любич Ю. Л. Конечномерный линейный анализ. М.: Наука, 1969.

[6] Любич Ю. Л. Линейный функциональный анализ // Итоги науки и техн. Соврем. пробл. матем. Фундамент. направления. Т. 19. М.: ВИНИТИ, 1988. С. 5-305.

[7] Данфорд Н., Шварц Дж. Линейные операторы. Спектральные операторы. М.: Мир, 1974.

[8] Хорн Р., Джонсон Ч. Матричный анализ. М.: Мир, 1989.

[9] Elsner L. On the variation of the spectra of matrices // Linear Algebra Appl. 1982. V. 47. P. 127-138.

Воронежский государственный технический университет

Поступило

Областной центр новых информационных технологий

17.05 .96

Воронежский государственный университет

E-mail : agyrya@vpicnit.voronezh.su 